\title{
Highly adherent small-colony variants of Pseudomonas aeruginosa in cystic fibrosis lung infection
}

Correspondence

Ivo Steinmetz

steinmetz.ivo@mh-hannover.de

Received 10 September 2002

Accepted 6 January 2003

\author{
Susanne Häußler, ${ }^{1}$ Isabell Ziegler, ${ }^{1}$ Alexandra Löttel, ${ }^{1}$ Franz v. Götz, ${ }^{1}$ \\ Manfred Rohde, ${ }^{2}$ Dirk Wehmhöhner, ${ }^{1}$ Selvan Saravanamuthu, ${ }^{1}$ \\ Burkhard Tümmler ${ }^{3}$ and Ivo Steinmetz ${ }^{1}$
}
${ }^{1,3}$ Institute of Medical Microbiology ${ }^{1}$ and Department of Pediatric Pneumology ${ }^{3}$, Hannover Medical School, Carl-Neuberg-Str. 1, 30625 Hannover, Germany

${ }^{2}$ German Research Centre for Biotechnology, Mascheroder Weg 1, 38124 Braunschweig, Germany

\begin{abstract}
Pseudomonas aeruginosa, an opportunistic human pathogen and ubiquitous environmental bacterium, is capable of forming specialized bacterial communities, referred to as biofilm. The results of this study demonstrate that the unique environment of the cystic fibrosis (CF) lung seems to select for a subgroup of autoaggregative and hyperpiliated $P$. aeruginosa small-colony variants (SCVs). These morphotypes showed increased fitness under stationary growth conditions in comparison with clonal wild-types and fast-growing revertants isolated from the SCV population in vitro. In accordance with the SCVs being hyperpiliated, they exhibited increased twitching motility and capacity for biofilm formation. In addition, the SCVs attached strongly to the pneumocytic cell line A549. The emergence of these highly adherent SCVs within the CF lung might play a key role in the pathogenesis of $P$. aeruginosa lung infection, where a biofilm mode of growth is thought to be responsible for persistent infection.
\end{abstract}

\section{INTRODUCTION}

Members of the species Pseudomonas aeruginosa are found in large numbers in many ecological niches of natural environments as well as in infections of humans, animals and plants. Since there seem to be no genetic or functional differences between environmental and clinical strains (Foght et al., 1996; Romling et al., 1994a), this universal distribution of $P$. aeruginosa indicates a remarkable degree of physiological and genetic versatility. The opportunistic pathogen $P$. aeruginosa is a key aetiological agent of hospital-acquired infections in the immunocompromised host and is responsible for chronic lung infection in cystic fibrosis (CF) patients (Gilligan, 1991; Lyczak et al., 2002; Govan \& Deretic, 1996; Breitenstein et al., 1997). Once CF patients become colonized by $P$. aeruginosa, there is a subsequent gradual deterioration in lung function, which determines the course and prognosis in most CF patients. Despite the fact that chronically infected CF patients harbour only one or few $P$. aeruginosa genotypes (Breitenstein et al., 1997), there is significant phenotypic variation in $P$. aeruginosa isolates from the CF lung, known as dissociative behaviour (Zierdt \& Schmidt, 1964). The chronically infected CF lung is thought to provide a habitat where $P$. aeruginosa in high cell densities faces a multiplicity of

Abbreviations: $\mathrm{CF}$, cystic fibrosis; SCV, small-colony variant. environmental challenges that lead to morphological diversification and the establishment of niche specialists (Oliver $e t$ al., 2000). Apart from the best-studied mucoid P. aeruginosa phenotype and other colony morphotypes (Govan \& Deretic, 1996), it has been recognized for many years that dwarf colonies can be isolated from the chronically infected respiratory tract of CF patients (Zierdt \& Schmidt, 1964). As shown recently in our laboratory, the recovery of dwarf or so-called small-colony variants (SCVs) of P. aeruginosa could be correlated with parameters revealing poor lung function and the use of inhaled antibiotics (Haussler et al., 1999). Recently, Deziel et al. (2001) isolated a hyperpiliated and highly adherent small phenotypic variant from a biofilm of $P$. aeruginosa strain 57RP in vitro. It was suggested that the occurrence of this variant within the ecological niche of the biofilm was regulated by a phase-variation mechanism. Similarly, a phase-variation mechanism has been proposed to be the cause of the phenotypic switch of an antibioticresistant and autoaggregative rough $\mathrm{SCV}$ of $P$. aeruginosa strain PA14 to wild-type revertants (Drenkard \& Ausubel, 2002). In this study, we identified a subgroup of hyperpiliated $P$. aeruginosa SCVs isolated from the respiratory tract of CF patients that exhibited similar phenotypic characteristics, as they showed autoaggregative growth behaviour in liquid cultures and increased biofilm formation capacity. Analysis of the phenotypic traits of the clinical SCV isolates in 
comparison with their revertants and clonal wild-types suggests that the appearance of the different morphotypes is not due to a variation between two phases but that mutation and selection might have been the driving forces of bacterial adaptation to given environmental conditions in the CF lung.

\section{METHODS}

Bacterial strains and culture conditions. Wild-type $P$. aeruginosa morphotypes and SCVs, which grew as characteristic small colonies, 1$3 \mathrm{~mm}$ in diameter (Haussler et al., 1999), were isolated from sputum samples or deep throat swabs of 12 CF patients who attended the CF clinic at Hannover Medical School, Germany. Fast-growing revertants were recovered from the SCV population after serial passages in brain heart infusion medium as described previously (Haussler et al., 1999). The SpeI -restriction profiles of SCV, wild-type and revertant DNA were shown to be identical using pulsed-field gel electrophoresis (Romling et al., 1994b), indicating clonal identity. Of the 12 P. aeruginosa strains with clonally identical SCVs, revertants and wild-types, the SCVs of six strains exhibited autoaggregative growth behaviour in liquid culture. This subgroup of SCVs, together with the respective wild-types and revertants, were chosen for further characterization. The sizes of single colonies of SCV, wild-type and revertant were assessed under a light microscope with an ocular micrometer.

Biofilm formation. A modified micro-titre plate test, as described by Stepanovic et al. (2000), was used to determine the biofilm formation capacity of SCVs in comparison with their corresponding revertants and wild-types. Briefly, bacteria grown overnight on Columbia agar plates were resuspended in modified Vogel-Bonner medium $(3.3 \mathrm{mM}$ $\mathrm{MgSO}_{4}, 10 \mathrm{mM}$ citric acid, $28 \mathrm{mM} \mathrm{NaNH}{ }_{4} \mathrm{HPO}_{4}, 37 \mathrm{mM} \mathrm{K} \mathrm{HPO}_{4}$, $214 \mathrm{mM}$ D-gluconic acid; $\mathrm{pH} 7 \cdot 4$ ). The $\mathrm{OD}_{650}$ of the bacterial suspensions was determined and aliquots of $100 \mu$, containing approximately $10^{7}$ bacteria, were inoculated in six parallel wells of a 96-well polystyrene plate. After incubation for $24 \mathrm{~h}$ at $37^{\circ} \mathrm{C}$, the wells were rinsed thoroughly with buffer A $(0.01 \mathrm{M}$ potassium phosphate buffer made isotonic with saline, $\mathrm{pH} 7 \cdot 5$ ) and then fixed with $150 \mu \mathrm{l}$ absolute methanol for $7 \mathrm{~min}$ at room temperature and the attached bacterial material was then stained by adding $150 \mu \mathrm{l}$ crystal violet $(1 \%$, $\mathrm{w} / \mathrm{v}$ ) for $30 \mathrm{~min}$. The plates were then rinsed with tap water and the amount of attached material was quantified by solubilization of the crystal violet dye in $150 \mu \mathrm{l} 33 \%$ glacial acetic acid. The $A_{620}$ was measured with the use of an ELISA reader.

Association with A549 cells. To assess the ability of SCVs, their revertants and wild-types not only to adhere to inanimate surfaces but also to associate with eukaryotic cells, the pneumocytic cell line A549 was used, obtained from the ATCC. For the assay, A549 cells were grown to confluence in Dulbecco's modified Eagle's medium containing $10 \%$ fetal calf serum at $37^{\circ} \mathrm{C}$ in an atmosphere of $5 \% \mathrm{CO}_{2}$, treated with trypsin/EDTA and diluted in fresh medium at a concentration of $2 \times 10^{5}$ cells $\mathrm{ml}^{-1}$.

The different bacterial morphotypes were grown overnight on Columbia agar plates and labelled with $10 \mu \mathrm{g}$ FITC $(\mathrm{mg} \text { wet weight bacteria })^{-1}$ in $200 \mu 150 \mathrm{mM}$ sodium carbonate buffer, $\mathrm{pH} 9 \cdot 8$. After $45 \mathrm{~min}$ at room temperature, the bacterial suspension was washed twice with Tyrode's solution (50 mM NaCl, $3 \mathrm{mM} \mathrm{KCl,} 50 \mathrm{mM}$ HEPES; Sigma) and resuspended in buffer A. The labelled bacteria were added to the A549 cells at an m.o.i. of one bacterium per two cells and incubated for $60 \mathrm{~min}$. After three washing steps with PBS ( $\mathrm{pH} 7 \cdot 2$ ), the cells were further analysed by flow cytometry using a Becton Dickinson FACScan.
Analysis of data was performed with CELL-Quest software (Becton Dickinson). Results are presented as the percentage of A549 cells associated with fluorescent bacteria.

Hydrophobicity assay. Estimation of cell-surface hydrophobicity of SCVs in comparison with their corresponding revertants and wild-types was performed using a standard MATH test (microbial adhesion to hydrocarbons) as described by Perez et al. (1998). Briefly, bacteria were grown to exponential phase in Luria-Bertani (LB) broth, washed with PBS and diluted to an $\mathrm{OD}_{600}$ of $0 \cdot 5$. An aliquot of $2 \mathrm{ml}$ of this suspension was mixed with $400 \mu \mathrm{l}$ xylene and vortexed for $120 \mathrm{~s}$. After $30 \mathrm{~min}$ of equilibration, the loss of optical density in the aqueous phase (OD) relative to that of the initial cell suspension $\left(\mathrm{OD}_{0}\right)$ was determined and hydrophobicity $(\mathrm{H} \%)$ was estimated by calculating the percentage of cells adhering to xylene with the formula $\mathrm{H} \%=\left[\left(\mathrm{OD}_{0}-\mathrm{OD}\right) / \mathrm{OD}_{0}\right]$ $\times 100$.

\section{Motility assays}

Swimming. The SCV, revertant and wild-type morphotypes were grown overnight on Columbia agar plates and inoculated with the use of a sterile toothpick onto swimming-agar plates containing $1 \%$ tryptone (Merck), $0.5 \% \mathrm{NaCl}$ and $0.3 \%$ agar. Motility was assayed after $48 \mathrm{~h}$ incubation at $30{ }^{\circ} \mathrm{C}$ by determining the radius of the circular expansion pattern of bacterial migration from the point of inoculation.

Twitching motility. The bacteria were stabbed through an agar layer of LB ( $1 \%$ agar) to the bottom of the Petri dish and incubated for $48 \mathrm{~h}$ at $37^{\circ} \mathrm{C}$. After removal of the agar, crystal violet $(1 \%, \mathrm{w} / \mathrm{v})$ was used to stain attached cells and the radius was determined for each morphotype. All motility assays were performed in triplicate.

Electron microscopy. For transmission electron microscopic analysis of negatively stained samples, bacteria were grown on twitchingmotility agar plates for $48 \mathrm{~h}$. Cells from the interface between agar and Petri dish were resuspended in TE buffer $(10 \mathrm{mM}$ Tris/ $\mathrm{HCl}, 2 \mathrm{mM}$ EDTA, pH 6.9) for about 1 min before a carbon-coated Formvar copper grid (300 mesh) was floated on the drop. The grid was rinsed with TE buffer and stained with a $2 \%$ aqueous solution of uranyl acetate, $\mathrm{pH} 4 \cdot 5$. Samples were examined in a Zeiss transmission electron microscope EM910 at an acceleration voltage of $80 \mathrm{kV}$.

RNA isolation and Northern blotting. Total RNA from $12 \mathrm{ml}$ bacterial culture in modified Vogel-Bonner medium $\left(0 \cdot 8 \times 10^{9}\right.$ cells $\mathrm{ml}^{-1}$ ) was extracted with a modified hot-phenol method (Oelmuller et al., 1990). Bacteria were harvested by centrifugation at $3800 \mathrm{~g}$ for $3 \mathrm{~min}$, resuspended in $0.5 \mathrm{ml}$ distilled water and lysed in $7.5 \mathrm{ml}$ phenol/ lysis buffer mix [ $5 \mathrm{ml}$ phenol (pH 5.5) plus $2.5 \mathrm{ml}$ lysis buffer ( $\%$ SDS, $30 \mathrm{mM}$ sodium acetate, $3 \mathrm{mM}$ EDTA, pH 5.5)] for $10 \mathrm{~min}$ with shaking. After centrifugation at $3800 \mathrm{~g}$ for $20 \mathrm{~min}$, the supernatant was extracted with $3 \mathrm{ml}$ phenol/chloroform (1:1) and subsequently with $3 \mathrm{ml}$ pure chloroform. To precipitate the nucleic acids, $0 \cdot 1$ vol. $3 \mathrm{M}$ sodium acetate $(\mathrm{pH} 5.2)$ and 2.5 vols ethanol were added and the sample was incubated at $-20{ }^{\circ} \mathrm{C}$ overnight and centrifuged for $30 \mathrm{~min}$ at $3800 \mathrm{~g}$. The pellet was washed with $5 \mathrm{ml} 70 \%$ ethanol and treated with 38 U DNase I (Roche) and $20 \mathrm{U}$ SUPERaseIn (Ambion) in DNase I buffer $(50 \mathrm{mM}$ sodium acetate, $10 \mathrm{mM} \mathrm{MgCl}_{2}, 2 \mathrm{mM} \mathrm{CaCl}_{2}$, pH 6.5) for $30 \mathrm{~min}$ at $37^{\circ} \mathrm{C}$ in a total volume of $200 \mu \mathrm{l}$. The yield of total cellular RNA was determined by measuring the $A_{260}$ after purification with RNeasy columns (Qiagen). Aliquots of $10 \mu \mathrm{g}$ RNA were separated electrophoretically in $1 \cdot 2 \%$ agarose with $2 \%$ formaldehyde as the denaturing reagent; the running buffer was $1 \times$ MOPS. Equal sample loadings were ascertained by densitometric analysis (PCBAS 2.09f; raytest) of the UV-induced fluorescence of the $23 \mathrm{~S}$ and $16 \mathrm{~S}$ rRNA bands. Immediately after electrophoresis, transfer of RNA to positively charged nylon membranes (Amersham) was done by capillary blotting with $20 \times \operatorname{SSC}(3 \mathrm{M} \mathrm{NaCl}$, $0 \cdot 3 \mathrm{M}$ sodium citrate, $\mathrm{pH} 7 \cdot 0)$ as transfer buffer. Genomic DNA was 
prepared from strain 20265 and a $643 \mathrm{bp}$ fragment of pilA was amplified by PCR as described by Spangenberg et al. (1995). Hybridization and detection of the digoxigenin-dUTP (Roche)-labelled probes were carried out as described elsewhere (Engler-Blum et al., 1993) with CDP-Star as the chemiluminescent substrate.

Relative fitness of the bacterial morphotypes. To estimate the capacity of the bacterial morphotypes to survive and reproduce under different environmental conditions, we determined the fitness of revertants and wild-types relative to SCVs in co-cultivation experiments (Lenski, 1991) in two experimental settings. Firstly, $5 \mathrm{ml} \mathrm{LB}$ broth was inoculated with approximately $10^{7}$ c.f.u. $\mathrm{ml}^{-1}$ of two bacterial morphotypes (SCVs and revertants or SCVs and wild-types) and incubated at $37^{\circ} \mathrm{C}$ with shaking. Every $24 \mathrm{~h}$, samples were collected and the change in frequency of colony morphotypes was determined over a 4-day period by plating diluted samples onto Columbia agar plates. Secondly, the relative fitness of the various morphotypes was tested after subculture in fresh medium. After $24 \mathrm{~h}, 50 \mu \mathrm{l}$ of the bacterial suspension was added to $50 \mathrm{ml}$ fresh LB broth and incubated for another $24 \mathrm{~h}$ at $37^{\circ} \mathrm{C}$ with shaking. The change in frequency of colony morphotypes was determined by plating serially diluted samples onto Columbia agar plates. All co-culture assays were performed in two independent experiments in duplicate. As a control, cultures of only SCVs, revertants or wild-types were grown under the conditions described and diluted samples were plated onto Columbia agar plates to determine the stability of the colony morphology.

\section{RESULTS}

\section{Growth behaviour of autoaggregative SCVs}

The phenotypic morphology of SCVs, revertants and wildtypes of the representative strain 20265 on Columbia agar plates is shown in Fig. 1(a). The wild-type P. aeruginosa strain formed irregularly shaped colonies, $\sim 3 \mathrm{~mm}$ in size. The SCV formed small ( $\sim 1 \mathrm{~mm}$ in diameter), convex, opaque and circular colonies after 2 days incubation at $37{ }^{\circ} \mathrm{C}$, whereas the revertants isolated after repeated subcultures were larger ( $\sim 10 \mathrm{~mm}$ in diameter) and flatter, with an irregular surface. In contrast to the wild-type and revertants, the six autoaggregative clinical SCV isolates did not grow homogeneously, dispersed in the medium, but formed stable, macroscopically visible clumps (Fig. 1b).

\section{Biofilm formation}

The six autoaggregative SCVs formed very dense and stable biofilms in non-agitated 96-well polystyrene ELISA plates (Fig. 2). Significantly less biofilm formation was observed for the corresponding clonal wild-type cultures (paired $t$-test; $P<0.001$ ) while, with the exception of one strain, the revertants exhibited an intermediate capacity for biofilm formation (Fig. 2).

\section{Association with A549 cells}

In order to determine the ability of the different bacterial morphotypes to associate with eukaryotic cells, we used the pneumocytic cell line A549 and performed a FACS analysis. Although the general capacity to associate with the cell line A549 was highly dependent on the strain tested, SCVs exhibited a significantly increased association with A549 cell surfaces compared with the wild-types (paired $t$-test; $P=0.02$ ) (Fig. 3).

\section{Surface hydrophobicity and pili}

By using a standard hydrophobicity assay (MATH test), the cell surfaces of the six autoaggregative SCV isolates were shown to be more hydrophobic than the corresponding wildtypes and revertants (paired $t$-test; $P=0.016$ ) (Fig. 4). We (a)

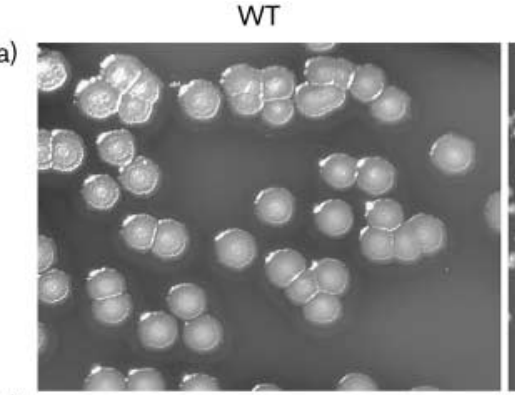

(b)

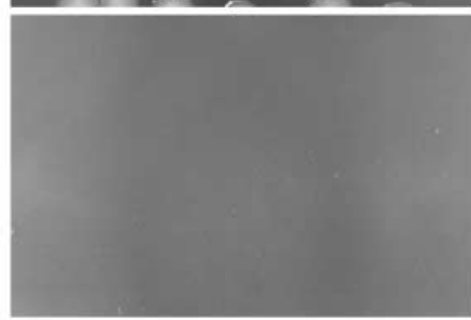

WT

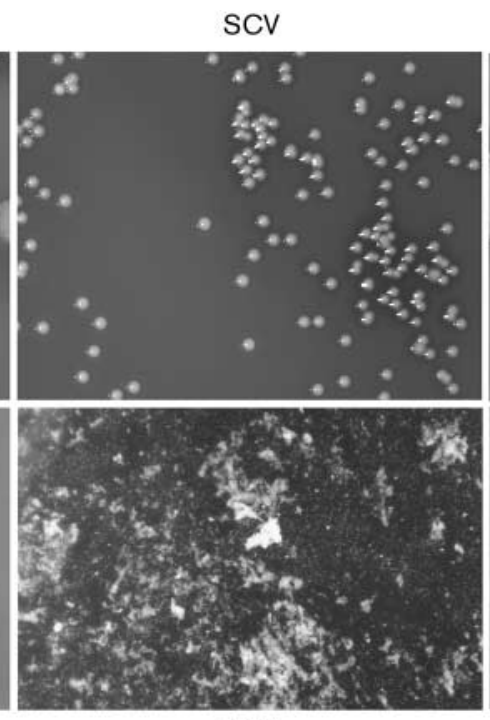

SCV

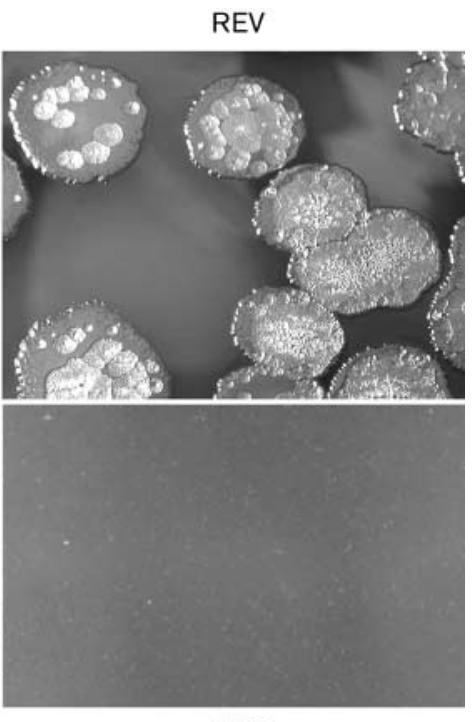

REV

Fig. 1. Growth of $P$. aeruginosa 20265 wild-type, SCV and revertant on agar plates and in liquid cultures. (a) Colony morphology of wild-type (WT) and clonally identical SCV and revertant (REV) of strain 20265 on Columbia agar plates incubated at $37^{\circ} \mathrm{C}$ for $48 \mathrm{~h}$.

(b) Autoaggregation of the SCV in comparison with the WT and REV in modified Vogel-Bonner medium grown for $24 \mathrm{~h}$. 


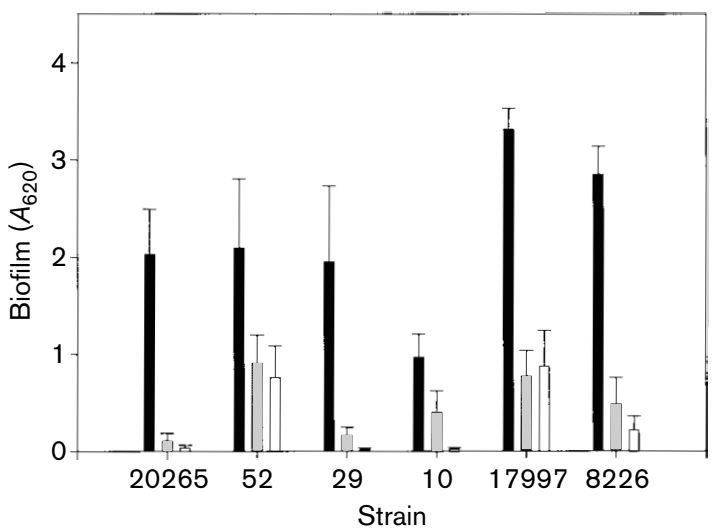

Fig. 2. Biofilm formation capacity of the autoaggregative SCV strains (filled bars) in comparison with their corresponding revertants (shaded bars) and wild-types (open bars) grown in microtitre plates. Data are means \pm SD of two independent experiments performed in six parallel wells.

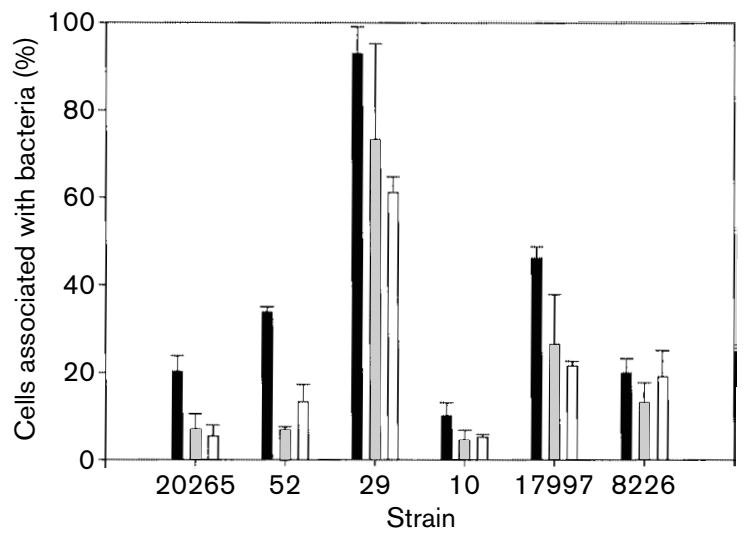

Fig. 3. Association of A549 cells with the autoaggregative SCV strains (filled bars) and their corresponding revertants (shaded bars) and wild-types (open bars). Data are means \pm SD of three independent experiments.

therefore speculated that the SCVs could be hyperpiliated, as has been shown for $P$. aeruginosa small phenotypic variants isolated from a biofilm in vitro (Deziel et al., 2001). Transmission electron microscopy of bacteria grown on twitching-agar plates for $48 \mathrm{~h}$ showed that, under the given growth conditions, five of the six highly adherent SCVs (with the exception of strain 17997) expressed abundant pili. The revertants tended to express even more pili (Fig. 5a), whereas no pilus structures were observed in four of the corresponding wild-types and only scant pili in the remaining two. As putative fimbrial adhesins have been suggested to be involved in biofilm formation of $P$. aeruginosa (Vallet et al., 2001), we performed a Northern blot analysis to confirm that the structures seen under the electron microscope were type IV pili. As shown in Fig. 5(b), the Northern blot revealed an

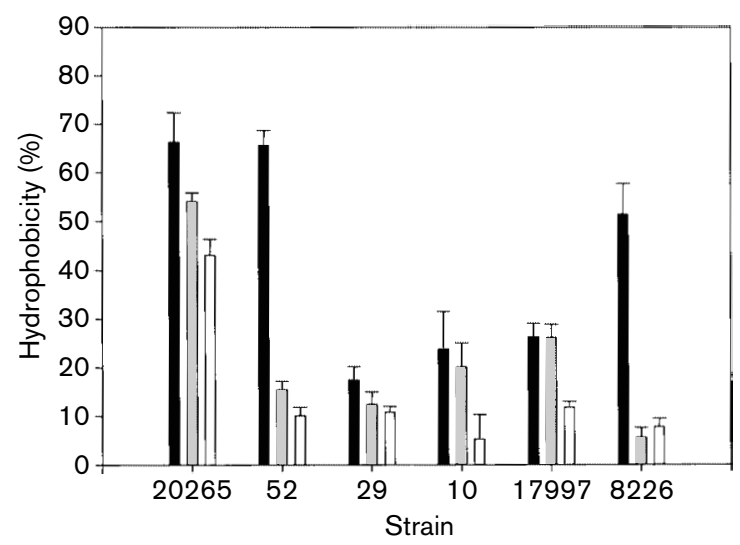

Fig. 4. Surface hydrophobicity of autoaggregative SCV strains (filled bars) and their corresponding revertants (shaded bars) and wild-types (open bars). Data are means \pm SD of three independent experiments.

increased level of pilA gene expression, encoding the structural subunit of type IV pili, in SCVs and revertants of strain 20265 in comparison with the corresponding wild-type.

\section{Swimming motility and twitching motility}

The reduced diameter of SCV colonies and the lack of an irregular-shaped surface suggested impaired motility of the SCVs in comparison with their revertants and wild-types. On swimming-agar plates, the six autoaggregative SCVs exhibited a reduced zone of motility within $48 \mathrm{~h}$ in comparison with wild-types and revertants. The mean radius of SCVs on swimming-agar plates was $0.93 \mathrm{~cm}$ (SD 0.59); the mean radii of the wild-type $(1.59 \pm 0.63 \mathrm{~cm}$; paired $t$-test, $P<0.001)$ and the revertants $(1.35 \pm 0.68 \mathrm{~cm}$; paired $t$-test, $P<0.001)$ were significantly larger.

As type IV pili are known to mediate twitching motility, we tested the six hyperpiliated SCVs in comparison with wildtypes and revertants for their twitching capacity. After stab inoculation through an LB agar layer, the SCV s formed larger zones of twitching motility at the agar-plastic interface (median radius $0.65 \mathrm{~cm}$; inner quartile $0.35-1.2$ ) compared with their corresponding wild-types (median radius $0.2 \mathrm{~cm}$; inner quartile $0 \cdot 1-0 \cdot 4$ ) (Wilcoxon signed rank test, $P<$ $0 \cdot 001$ ), whereas the revertants exhibited the largest zones of twitching motility (median radius $0.95 \mathrm{~cm}$; inner quartile $0 \cdot 7-1.75$ ) (Wilcoxon signed rank test, $P<0.001$ ).

\section{Fitness of SCVs}

To estimate the fitness of autoaggregative SCVs under late stationary growth phase conditions relative to the corresponding wild-types and revertants, co-culture experiments were performed in LB broth. In contrast to the intensive clumping of SCVs in modified Vogel-Bonner medium, SCVs exhibited far less autoaggregative growth behaviour when cultured in LB broth. After 1 day in co-culture, the numbers of c.f.u. of the six wild-types exceeded the counts for SCVs, depending on the strain tested, by approximately 2 - to 
(a)
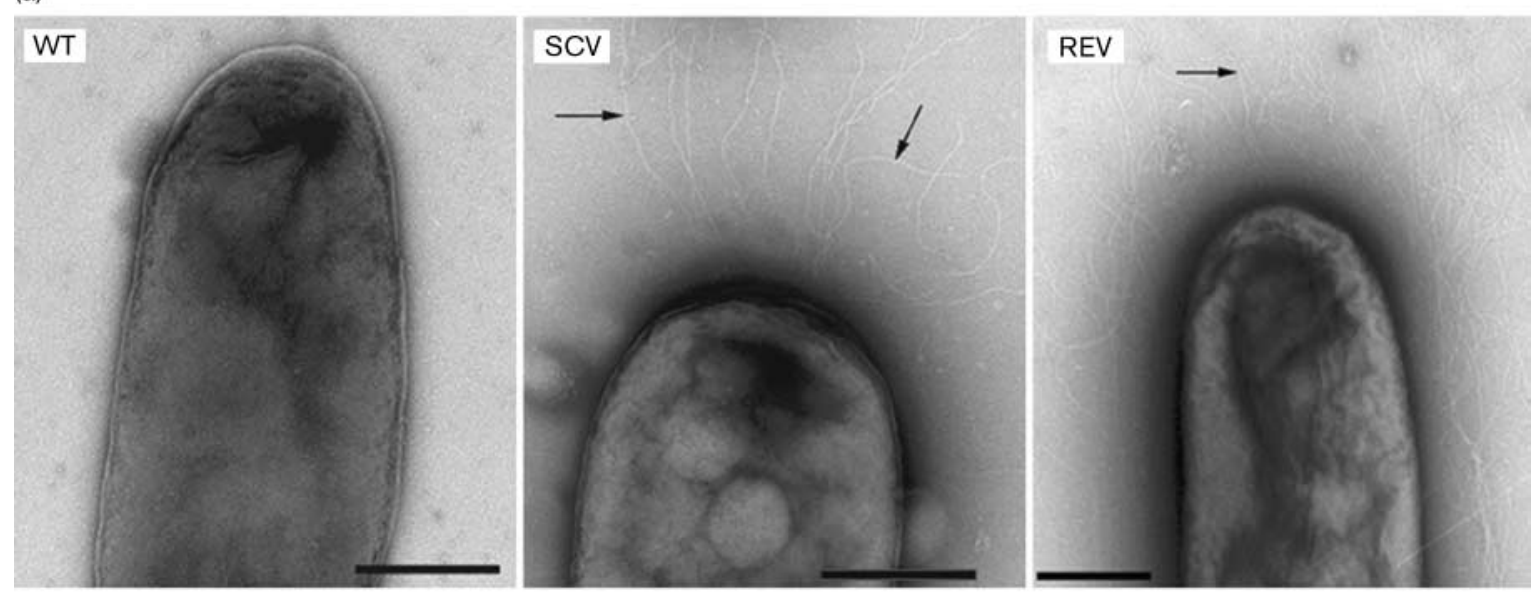

(b)

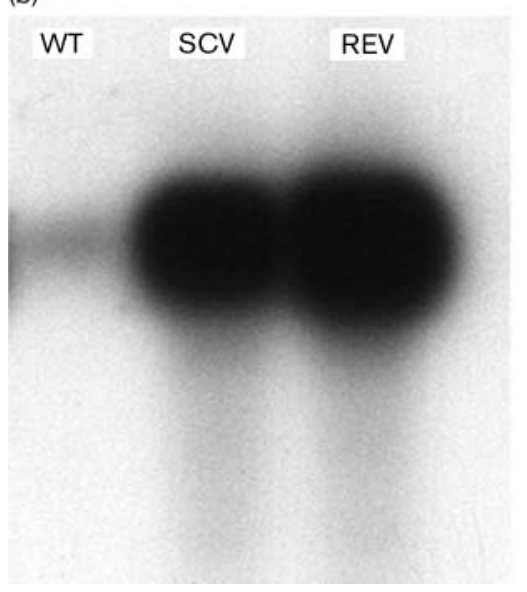

10-fold. However, with the exception of one strain (strain 10 ), prolonged incubation (4 days) led to a change of relative fitness in favour of the SCVs. In comparison to day 1, the ratio of numbers of c.f.u. of SCVs versus wild-types increased in the range of approximately 2 - to 80 -fold. Fig. 6 shows the selective advantage of SCVs of strain 20265 compared with

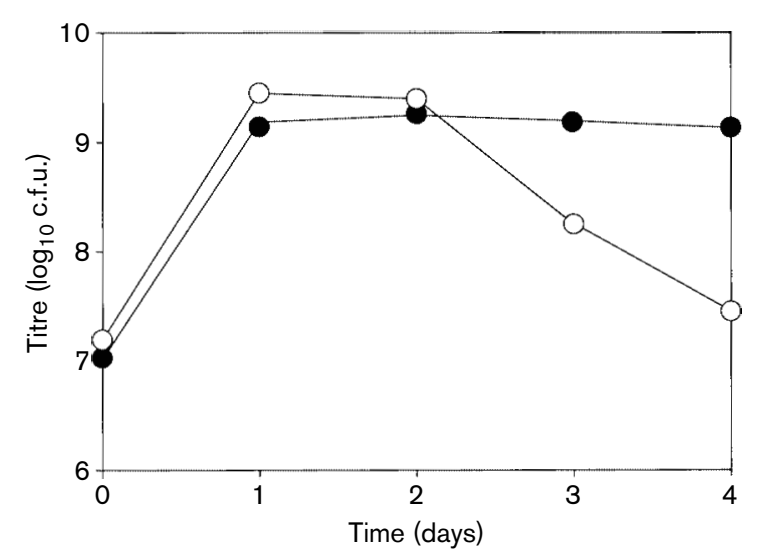

Fig. 6. Relative fitness of $P$. aeruginosa 20265 wild-type (o) versus $\operatorname{SCV}(\bullet)$.
Fig. 5. Transmission electron micrographs (a) and Northern blot analysis of pilA transcription (b) of $P$. aeruginosa 20265 wild-type (WT), SCV and revertant (REV). Bars, $1 \mu \mathrm{m}$.

the wild-type after prolonged incubation. In this strain, the increased fitness of the SCVs versus their corresponding wild-types was particularly pronounced. After 4 days of growth, the number of SCVs of strain 20265 exceeded not only the number of wild-types but also the number of revertants, although to a lesser extent (data not shown).

In contrast to these results, experiments with strain 20265 in which co-cultures were passaged into fresh medium after $24 \mathrm{~h}$ revealed that, after $48 \mathrm{~h}$, revertants exceeded the SCVs by $15 \cdot 1$-fold (SD 7.2), while the number of wild-types exceeded the SCVs by $10 \cdot 1$-fold (SD 5.5). Pure cultures of SCVs, revertants and wild-types that were grown under the conditions described as a control did not reveal any changes in colony morphology.

\section{DISCUSSION}

The morphological diversity of $P$. aeruginosa recovered from the respiratory tract material of CF patients is well known (Zierdt \& Schmidt, 1964) and has been suggested to be almost pathognomonic for the chronically infected CF lung (Govan \& Deretic, 1996). Those phenotypic variants that can face the challenges of the heterogeneous and changing habitat imposed by antibiotic therapies and the host immune 
system dominate in clonal bacterial populations (Oliver et al., 2000). Most obviously, during chronic debilitating pulmonary infection, there is a subsequent emergence of mucoid P. aeruginosa phenotypes (Govan \& Deretic, 1996). Although studies have so far been focussed on the most common mucoid phenotype, other phenotypic variants do exist in the chronically infected CF lung, e.g. so-called SCVs, comprising phenotypic variants characterized by slow growth on agar plates and increased antibiotic resistance against a broad range of antipseudomonal agents (Haussler $e t$ al., 1999). Apart from the antibiotic selection pressure, which might favour the occurrence of SCVs, this study demonstrates that the respiratory tract of CF patients obviously provides a niche for a subgroup of autoaggregative and highly adherent $P$. aeruginosa SCVs. $P$. aeruginosa has been shown to persist within a biofilm in the CF lung (Lam et al., 1980; Worlitzsch et al., 2002), which is the prevailing microbial lifestyle in most natural environments. The importance of the biofilm mode of growth in the context of chronic infections is gaining increasing attention (Costerton et al., 1995, 1999; O'Toole et al., 2000). In vitro studies on biofilms have shown that their formation proceeds through distinct developmental steps (O’Toole \& Kolter, 1998), beginning with the approach of bacteria to the surface, slowing of motility (Garrett et al., 1999) and attachment of single cells to the surface and/or other microbes previously attached. These initial steps are followed by movement of the bacteria on the surface to form clumps of cells or microcolonies, referred to as twitching motility (O'Toole \& Kolter, 1998; Doig et al., 1988). This motility is believed to be mediated by the extension and contraction of type IV pili (Darzins, 1994; Wall \& Kaiser, 1999). Finally, exopolysaccharides are produced to form three-dimensional biofilms and stabilize them (Watnick \& Kolter, 2000; Hentzer et al., 2001). The increased capacity of hyperpiliated $P$. aeruginosa SCVs described in this study to form biofilms and to attach to host cells and to each other might be considered as major virulence traits that are selected for in the CF lung. Type IV pili have been shown to be important for the adherence to and colonization of eukaryotic cell surfaces, and are thought to play a role in pathogenesis (Doig et al., 1988; Bieber et al., 1998; Comolli et al., 1999).

Not only adherent growth characteristics but also slight differences in the ability to exploit resources or to escape adversity may favour the persistence of phenotypic variants. As demonstrated in co-cultivation experiments, the highly adherent SCVs seemed to have a selective advantage in the late stationary phase of liquid cultures. Thus, limited nutrient conditions may favour growth of SCVs in the CF lung. As opposed to the growth advantage of SCVs in stationary cultures, the fast-growing phenotypes (wild-types and revertants) dominated under exponential phase growth conditions. The phenotypic diversity of our clinical morphotypes did not seem to be due to a variation between two phases, as has been proposed for the P. aeruginosa 57RP small phenotypic variant isolated from a biofilm in vitro (Deziel et al., 2001) and for the autoaggregative SCVs of $P$. aeruginosa strain PA14 (Drenkard \& Ausubel, 2002). Analysis of several phenotypic traits of our clinical isolates revealed that revertants varied from wild-types and, although fast-growing (Haussler et al., 1999), still had properties of SCVs such as increased biofilm formation and attachment to cell surfaces as well as the retained expression of abundant pili compared with wild-types. We therefore propose that the diversity of our $P$. aeruginosa morphotypes could be due to adaptive mutations and selection, as has been described for the morphological diversity of Pseudomonas fluorescens phenotypic variants in the heterogeneous environment of a static broth (Rainey \& Travisano, 1998). Future studies will have to elucidate the molecular basis for the emergence of highly adherent, autoaggregative and hyperpiliated $P$. aeruginosa variants within the $\mathrm{CF}$ lung and their possible role in the process of biofilm formation and thus their clinical significance.

\section{REFERENCES}

Bieber, D., Ramer, S. W., Wu, C. Y., Murray, W. J., Tobe, T., Fernandez, R. \& Schoolnik, G. K. (1998). Type IV pili, transient bacterial aggregates, and virulence of enteropathogenic Escherichia coli. Science 280, 2114-2118.

Breitenstein, S., Walter, S., Bosshammer, J., Romling, U. \& Tummler, B. (1997). Direct sputum analysis of Pseudomonas aeruginosa macrorestriction fragment genotypes in patients with cystic fibrosis. Med Microbiol Immunol 186, 93-99.

Comolli, J. C., Hauser, A. R., Waite, L., Whitchurch, C. B., Mattick, J. S. \& Engel, J. N. (1999). Pseudomonas aeruginosa gene products PilT and PilU are required for cytotoxicity in vitro and virulence in a mouse model of acute pneumonia. Infect Immun 67, 3625-3630.

Costerton, J. W., Lewandowski, Z., Caldwell, D. E., Korber, D. R. \& Lappin-Scott, H. M. (1995). Microbial biofilms. Annu Rev Microbiol 49, 711-745.

Costerton, J. W., Stewart, P. S. \& Greenberg, E. P. (1999). Bacterial biofilms: a common cause of persistent infections. Science 284, $1318-1322$.

Darzins, A. (1994). Characterization of a Pseudomonas aeruginosa gene cluster involved in pilus biosynthesis and twitching motility: sequence similarity to the chemotaxis proteins of enterics and the gliding bacterium Myxococcus xanthus. Mol Microbiol 11, 137-153.

Deziel, E., Comeau, Y. \& Villemur, R. (2001). Initiation of biofilm formation by Pseudomonas aeruginosa 57RP correlates with emergence of hyperpiliated and highly adherent phenotypic variants deficient in swimming, swarming, and twitching motilities. J Bacteriol 183, 1195-1204.

Doig, P., Todd, T., Sastry, P. A., Lee, K. K., Hodges, R. S., Paranchych, W. \& Irvin, R. T. (1988). Role of pili in adhesion of Pseudomonas aeruginosa to human respiratory epithelial cells. Infect Immun 56, 1641-1646.

Drenkard, E. \& Ausubel, F. M. (2002). Pseudomonas biofilm formation and antibiotic resistance are linked to phenotypic variation. Nature 416, 740-743.

Engler-Blum, G., Meier, M., Frank, J. \& Muller, G. A. (1993). Reduction of background problems in nonradioactive northern and Southern blot analyses enables higher sensitivity than ${ }^{32} \mathrm{P}$-based hybridizations. Anal Biochem 210, 235-244.

Foght, J. M., Westlake, D. W., Johnson, W. M. \& Ridgway, H. F. (1996). Environmental gasoline-utilizing isolates and clinical isolates of Pseudomonas aeruginosa are taxonomically indistinguishable by chemotaxonomic and molecular techniques. Microbiology 142, 2333-2340. 
Garrett, E. S., Perlegas, D. \& Wozniak, D. J. (1999). Negative control of flagellum synthesis in Pseudomonas aeruginosa is modulated by the alternative sigma factor AlgT (AlgU). J Bacteriol 181, 7401-7404.

Gilligan, P. H. (1991). Microbiology of airway disease in patients with cystic fibrosis. Clin Microbiol Rev 4, 35-51.

Govan, J. R. \& Deretic, V. (1996). Microbial pathogenesis in cystic fibrosis: mucoid Pseudomonas aeruginosa and Burkholderia cepacia. Microbiol Rev 60, 539-574.

Haussler, S., Tummler, B., Weissbrodt, H., Rohde, M. \& Steinmetz, I. (1999). Small-colony variants of Pseudomonas aeruginosa in cystic fibrosis. Clin Infect Dis 29, 621-625.

Hentzer, M., Teitzel, G. M., Balzer, G. J., Heydorn, A., Molin, S., Givskov, M. \& Parsek, M. R. (2001). Alginate overproduction affects Pseudomonas aeruginosa biofilm structure and function. J Bacteriol 183, 5395-5401.

Lam, J., Chan, R., Lam, K. \& Costerton, J. W. (1980). Production of mucoid microcolonies by Pseudomonas aeruginosa within infected lungs in cystic fibrosis. Infect Immun 28, 546-556.

Lenski, R. E. (1991). Quantifying fitness and gene stability in microorganisms. Biotechnology 15, 173-192.

Lyczak, J. B., Cannon, C. L. \& Pier, G. B. (2002). Lung infections associated with cystic fibrosis. Clin Microbiol Rev 15, 194-222.

Oelmuller, U., Schlegel, H. G. \& Friedrich, C. G. (1990). Differential stability of mRNA species of Alcaligenes eutrophus soluble and particulate hydrogenases. J Bacteriol 172, 7057-7064.

Oliver, A., Canton, R., Campo, P., Baquero, F. \& Blazquez, J. (2000). High frequency of hypermutable Pseudomonas aeruginosa in cystic fibrosis lung infection. Science 288, 1251-1254.

O'Toole, G. A. \& Kolter, R. (1998). Flagellar and twitching motility are necessary for Pseudomonas aeruginosa biofilm development. Mol Microbiol 30, 295-304.

O'Toole, G., Kaplan, H. B. \& Kolter, R. (2000). Biofilm formation as microbial development. Annu Rev Microbiol 54, 49-79.
Perez, P. F., Minnaard, Y., Disalvo, E. A. \& De Antoni, G. L. (1998). Surface properties of bifidobacterial strains of human origin. Appl Environ Microbiol 64, 21-26.

Rainey, P. B. \& Travisano, M. (1998). Adaptive radiation in a heterogeneous environment. Nature 394, 69-72.

Romling, U., Wingender, J., Muller, H. \& Tummler, B. (1994a). A major Pseudomonas aeruginosa clone common to patients and aquatic habitats. Appl Environ Microbiol 60, 1734-1738.

Romling, U., Fiedler, B., Bosshammer, J., Grothues, D., Greipel, J., von der Hardt, H. \& Tummler, B. (1994b). Epidemiology of chronic Pseudomonas aeruginosa infections in cystic fibrosis. J Infect Dis 170, 1616-1621.

Spangenberg, C., Fislage, R., Sierralta, W., Tummler, B. \& Romling, U. (1995). Comparison of type IV-pilin genes of Pseudomonas aeruginosa of various habitats has uncovered a novel unusual sequence. FEMS Microbiol Lett 125, 265-273.

Stepanovic, S., Vukovic, D., Dakic, I., Savic, B. \& Svabic-Vlahovic, M. (2000). A modified microtiter-plate test for quantification of staphylococcal biofilm formation. J Microbiol Methods 40, 175-179.

Vallet, I., Olson, J. W., Lory, S., Lazdunski, A. \& Filloux, A. (2001). The chaperone/usher pathways of Pseudomonas aeruginosa: identification of fimbrial gene clusters ( cup) and their involvement in biofilm formation. Proc Natl Acad Sci U S A 98, 6911-6916.

Wall, D. \& Kaiser, D. (1999). Type IV pili and cell motility. Mol Microbiol 32, $1-10$.

Watnick, P. \& Kolter, R. (2000). Biofilm, city of microbes. J Bacteriol 182, 2675-2679.

Worlitzsch, D., Tarran, R., Ulrich, M. \& 12 other authors (2002). Effects of reduced mucus oxygen concentration in airway Pseudomonas infections of cystic fibrosis patients. J Clin Invest 109, 317-325.

Zierdt, C. H. \& Schmidt, P. J. (1964). Dissociation in Pseudomonas aeruginosa. J Bacteriol 87, 1003-1010. 